\title{
Alltagsaktivität und zirkadiane Variabilität ventrikulärer Tachyarrhythmien bei Patienten mit implantiertem Defibrillator
}

\author{
R. Fries ${ }^{1}$, J. König ${ }^{2}$, O. Schonecke ${ }^{3}$, H.-J. Schäfers ${ }^{4}$, M. Böhm ${ }^{1}$ \\ 1 Medizinische Klinik und Poliklinik, Innere Medizin III , Kardiologie/Angiologie (Direktor: Prof. Dr. M. Böhm) \\ 2 Institut für Medizinische Biometrie, Epidemiologie und Informatik (Direktor: Prof. Dr. U. Feldmann) \\ 3 Institut für Medizinische und klinische Psychologie (Ehem. Direktor: Prof. Dr. O. Schonecke†) \\ 4 Abteilung für Thorax- und Herzgefäßchirurgie (Direktor: Prof. Dr. H.J. Schäfers), Universitätskliniken des Saarlandes, Homburg/Saar
}

Hintergrund und Fragestellung: Akute kardiovaskuläre Krankheitsereignisse können durch körperliche und mentale Belastungen getriggert werden. Korreliert demnach auch die typische zirkadiane Variation von Arrhythmierezidiven bei Defibrillatorträgern (morgendliche Ereignishäufung) mit der individuellen Verteilung gewohnter physischer und mentaler Aktivitäten im Alltag?

Patienten und Methodilk: Bei 29 Patienten mit Arrhythmierezidiven an mindestens 3 Tagen nach Defibrillatorimplantation wurde die Verteilung gewohnter Aktivitäten mit Hilfe eines neu entwickelten Fragebogens in einem ärztlichen Interview erfasst, graduiert und mit der individuellen zirkadianen Ereignisverteilung im Langzeitverlauf korreliert.

Ergebnisse: Das höchste Niveau physischer bzw. mentaler Aktivitäten fand sich bei $55 \%$ bzw. 23\% der Patienten zur Tageszeit mit den meisten Rezidiven. Ein Vergleich des Aktivitätsniveaus in der Tagesperiode mit den häufigsten Ereignissen und dem mittleren Aktivitätsniveau in den übrigen Tagesperioden (ohne Schlafzeit) ergab signifikant höhere Werte für die physische Aktivität in dem Tageszeitraum mit individuellem Ereignisgipfel. Es bestand eine positive Korrelation zwischen der tageszeitlichen Variation der Arrhythmieinzidenz und der Summe aller Aktivitäten $(r=0,34, p<0,05)$ sowie der physischen Aktivitäten allein $(r=0,25, p=0,056)$, wohingegen ein Zusammenhang mit nur der mentalen Aktivitätsverteilung nicht aufgezeigt werden konnte $(r=0,02, p=0,84)$.

Schlussfolgerung: Die tageszeitliche Variation von kardiovaskulären Krankheitsereignissen kann als maßgeblicher Einflussfaktor auf die habituelle tageszeitliche Verteilung von Alltagsaktivitäten diskutiert werden.

\section{Daily activities and circadian variation of ventricular tachyarrhythmias in patients with implanted defibrillator}

Background and study aim: Acute cardiovascular events may be triggered by physical and mental stress. Does also the typical circadian variation of arrhythmia recurrence (morning peak of events) in patients with implanted cardioverter-defibrillator correlate with the individual circadian variation of physical and mental daily activities?

Patients and methods: In 29 consecutive patients with a minimum of three documented ventricular tachyarrhythmias after defibrillator implantation the circadian variation of habitual daily activities was determined in a standardized interview and compared to the individual circadian variation of arrhythmic events saved in the defibrillator event memory during long-term follow-up.

Results: The habitually highest physical and mental activity was determined during the time period with most arrhythmic events in $55 \%$ and $23 \%$ of patients, respectively. A comparison of the level of activity during the time period with the highest event frequency to the mean activity level during the rest of the day (without sleeping time) revealed significantly higher physical activity during the time of day with the highest arrhythmia recurrence. There was a positive correlation between circadian variation of arrhythmic events and the sum of all activities $(r=0.34, p<0.05)$ and physical activities alone $(r=0.25, p=0.056)$ while a relationship to mental activities alone could not be shown $(r=0.02, p=0.84)$

Conclusion: The circadian variation of acute cardiovascular events may be discussed as an essential factor influencing the circadian variation of habitual daily activities in patients with cardiac disease.
In zahlreichen Untersuchungen konnte die Triggerung akuter kardiovaskulärer Krankheitsereignisse durch körperliche und mentale Belastungen belegt werden. Dies gilt nicht nur für transiente Moykardischämien oder Infarkte $(2,4,5,8,11,12,14,15,16,17,24,25,26,27)$, sondern auch für den zum großen Teil durch spontane ventrikuläre Tachyarrhythmien bedingten (3) plötzlichen Herztod (7,9,21,22,23,27). Als potenzielle Triggerfaktoren wurden hierbei nicht nur Anstrengungen besonders hohen Ausmaßes gefunden, sondern auch geringere Belastungen, die innerhalb der individuellen Lebensroutine vieler Menschen zu erwarten sind $(2,4,5,8,11,12,14,16,17)$. Erste Untersuchungen, die in diesem Zusammenhang die rhythmologischen Speicherdaten moderner Defibrillatoren auswerteten, weisen darauf hin, dass auch die Anzahl der therapiebedürftigen Arrhythmierezidive bei Patienten mit implantierbarem

Dtsch Med Wochenschr 2001; 126: 1385-1390

(c) Georg Thieme Verlag Stuttgart · New York
Defibrillator durch physische und psychische Belastungen beeinflusst sind (unpublizierte eigene Daten,18,19). Vorläufigen Mitteilungen zufolge ist das Risiko für die Notwendigkeit einer Schockabgabe unter körperlicher und psychischer Belastung erhöht (19), und das Ausmaß der psychischen Belastung von Defibrillatorträgern hängt mit der Anzahl der notwendigen Therapieabgaben im Kurzzeitverlauf zusammen (18).

Es stellt sich also die Frage, ob sich die angesprochenen Zusammenhänge zwischen individueller Belastung und Rezidivhäufigkeit in der individuellen zirkadiane Variation der Rezidivarrhythmien bei Defibrillatorträgern widerspiegeln und damit, ob die bekannte zirkadiane Variabilität von Arrhythmien mit typischer morgendlicher Ereignishäufung $(13,20,28)$ nicht maßgeblich durch die gewohnheitsmäßige Aktivitätsverteilung der Patienten bestimmt wird. $\mathrm{Zu}$ dieser Fragestellung liegen bisher keine Daten vor. Ziele der vorliegenden Studie bei Patienten mit implantierbarem Defibrillator waren daher 1. 
eine Analyse der individuellen tageszeitlichen Verteilung der gewohnten physischen und mentalen Aktivitäten im täglichen Leben und 2. die Untersuchung des potenziellen Einflusses auf die zirkadiane Verteilung der Arrhythmierezidive.

\section{Patienten und Methoden}

\section{Patienten}

Konsekutiv untersucht wurden 29 Patienten nach Defibrillatorimplantation, bei denen anhand von mindestens 3 Tagen mit dokumentierten Rezidivarrhythmien im Langzeitverlauf eine statistisch sinnvolle Aussage über die zirkadiane Variabilität ihrer Rezidivverteilung gemacht werden konnte. Die untersuchten Patienten sind Teil aller 225 Patienten, die zwischen 1989 und 1998 in den Universitätskliniken Homburg mit einem implantierbaren Defibrillator versorgt wurden. 101 Patienten hatten bis zum Beginn der Untersuchung bereits spontane Arrhythmierezidive erlebt. Bei 83 dieser Patienten war die Episodendokumentation vollständig, und 52 Patienten hatten zudem Ereignisse an mindestens 3 Tagen der Nachbeobachtung. Von diesen standen zum Untersuchungszeitpunkt 29 zur Verfügung. Es handelte sich um 25 Männer (86\%) und vier Frauen (14\%) im Alter von 59 \pm 13 Jahren. 23 Patienten (79\%) hatten eine koronare Herzkrankheit, vier (14\%) eine dilatative Kardiomyopathie und zwei (7\%) andere kardiale Erkrankungen. Die linksventrikuläre Auswurffraktion war signifikant reduziert (LVEF $36 \pm 13 \%$, nur zwei Patienten mit normaler linksventrikulärer Funktion), wobei sich zwei Patienten (7\%) im Herzinsuffizienzstadium NYHA I, 17 (59\%) im NYHA-Stadium II und zehn Patienten (34\%) im NYHAStadium III befanden. Die Indikation zum Defibrillatoreinbau war bei fünf Patienten (17\%) das Überleben von Kammerflimmern, bei elf Patienten (38\%) die Dokumentation von hämodynamisch kompromittierenden Kammertachykardien und bei 13 Patienten (45\%) das Auftreten beider Arrhythmien. 22 Patienten (76\%) standen unter ACE-Hemmertherapie, und 19 Patienten (66\%) wurden zusätzlich mit einem Diuretikum behandelt. Zehn Patienten (34\%) standen von Beginn bis Ende der Nachbeobachtung ununterbrochen und vier Patienten (14\%) zeitweise unter Amiodaron; 19 Patienten (66\%) erhielten ununterbrochen und fünf Patienten (17\%) zeitweise Betablocker. Nur ein Patient wurde weder mit Betablocker noch mit Amiodaron behandelt. Andere Antiarrhythmika wurden nicht eingesetzt.

\section{Methoden}

Durch die Speicherfunktionen moderner Defibrillatoren werden spontane Arrhythmierezidive mit Datum, Uhrzeit und EKG-Beispielen registriert und können anschließend kritisch bewertet werden. In die Studie eingeschlossen wurden nur Patienten mit vollständiger Arrhythmiedokumentation. Da eine implantationsbedingte Arrhythmiehäufung in den ersten Wochen nach dem Geräteeinbau bekannt ist, wurden nur Rezidive ab der 5 . Woche nach Implantation analysiert. Berücksichtigt wurden nur adäquat detektierte und durch Schockabgabe oder antitachykardes Pacing therapierte ventrikuläre Tachyarrhythmien. Bei fünf Patienten wurden fehldetektierte Episoden von Sinustachykardien bzw. Vorhofflimmern dokumentiert und ausgeschlossen. In keinem Fall konnten reversible Ursachen für Rezidive wie instabile Koronarsyndrome oder Elektrolytentgleisungen wahrscheinlich gemacht werden. Die Ermittlung der tageszeitlichen Rezidivverteilung erfolgte durch Mittelung der prozentualen Arrhythmieverteilung an den einzelnen Ereignistagen der Patienten nach Grup- pierung der Ereignisse in vier prospektiv definierte Tageszeitzonen (Periode 1:0 Uhr bis 5 Uhr 59, 2:6 Uhr bis 11 Uhr 59, $3: 12$ Uhr bis 17 Uhr 59 und 4:18 Uhr bis 23 Uhr 59).

Auf der Basis von validierten Verfahren zur quantitativen Erfassung der Belastung durch Alltagsaktivitäten $(1,14)$ wurde in Zusammenarbeit mit der Abteilung für klinische Psychologie (Ehem. Direktor Prof. Dr. O. Schonecke†) der Universitätskliniken des Saarlandes ein Fragebogen entworfen, mit dessen Hilfe in einem ärztlich geführten Patienteninterview Informationen zur tageszeitlichen Verteilung physischer und mentaler Alltagsbelastungen erfasst werden können. Den Kategorien »überwiegend physische oder mentale Aktivität» wurden die folgenden Bereiche zugeordnet.

Physische Aktivitäten: Berufliche Tätigkeiten, Einkauf/Besorgungen, Hausarbeit, Heimwerken/Gartenarbeit, außerhäusliche soziale Aktivitäten, Spaziergänge, Treppensteigen, Sport, andere.

Die zur allgemeinen Lebensführung notwendigen Basistätigkeiten wie Umkleiden, Körperpflege, Nahrungsaufnahme und Toilettengang wurden nicht bewertet, da 1. ihre Durchführung für jedermann gleichermaßen erforderlich ist, 2. eine relevante körperliche Belastung hiervon nur in Ausnahmefällen ausgeht, und 3. bedeutsame Unterschiede in der tageszeitlichen Verteilung dieser Handlungen in einer Patientengruppe aus demselben Kulturkreis nicht zu erwarten sind.

Mentale Aktivitäten: Berufliche Tätigkeiten, Lesen, Fernsehen/Radio/Musikhören, Gesprächskontakte, Gesellschaftsspiele, Computer-/Schreib-/Verwaltungsarbeit, Musizieren, Handarbeit, Autofahren, andere.

Die zu erwartende Belastung wurde gemäß den Patientenangaben für jede der oben genannten Tageszeitperioden (morgens/mittags/abends/nachts) nach den folgenden Kriterien kategorisiert:

(1) Häufigkeit I - III:

$\mathrm{I}=$ selten (weniger als einmal pro Woche)

$\mathrm{II}=$ oft (mindestens 1 mal pro Woche)

III = täglich

(2) Intensität I - III:

\section{Körperliche Aktivitäten}

I = Tätigkeit wird als körperlich nicht anstrengend empfunden II = Tätigkeit wird als körperlich anstrengend empfunden III = durch die Tätigkeit bedingte Atemnot und/oder Schwitzen

\section{Mentale Aktivitäten}

I = Tätigkeit wird nebenbei ausgeführt

II = Patient konzentriert sich auf die Tätigkeit

III = Patient ist während der Tätigkeit hochkonzentriert

Auf eine Bewertung der Aktivitätsdauer wurde bewusst verzichtet, da einerseits davon auszugehen ist, dass eine Belastung durch Aktivität auch noch eine unbestimmte Zeit nach Belastungsende auf den Organismus einwirkt, wodurch sich kurze Belastungen in ihrer Bedeutung längerandauernden Belastun- 
gen annähern. Andererseits ist zu befürchten, dass die Mehrzahl der Patienten durch zusätzliche Fragen nach der tageszeitlichen Varianz der Dauer von Alltagsaktivitäten überfordert wären.

Die Angaben zu den Alltagsaktivitäten wurden zur Analyse der tageszeitlichen Belastungsvariation nach einem Punktesystem wie folgt kodiert.

Häufigkeit: I= 0,5 Punkte, II = 1 Punkt, III = 3 Punkte

Intensität:

I = unmultiplizierte Punktesumme,

II = Multiplikation mit 2,

III = Multiplikation mit 3

\section{Statistik}

Zur Klärung eines potenziellen Zusammenhangs der habituellen zirkadianen Aktivitätsverteilung mit der Rezidivverteilung im Langzeitverlauf wurden die Ergebnisse auf zwei Wegen statistisch untersucht:

1. Die Anzahl an Aktivitätspunkten in der Tageszeitperiode mit Ereignisgipfel wurde mit der mittleren Punktesumme in den übrigen Tageszeitperioden verglichen und mit Hilfe eines Mann-Whitney U-Tests bzw. Wilcoxon Signed Rank Tests auf Signifikanz überprüft. 2. Die pro Tageszeitperiode nach physischer und mentaler Aktivität getrennt errechnete Punktesumme wurde für jeden Patienten in Prozentangaben umgerechnet und mit den entsprechenden Prozentangaben zur tageszeitlichen Rezidivverteilung linear korreliert. Die 29 Steigungswerte der individuellen Regressionsanalysen wurden anschließend in einem Wilcoxon Signed Rank Test gegen Null auf Signifikanz geprüft.

Für die stetigen Parameter wurde eine Varianzanalyse (ANOVA) durchgeführt, wobei die Parameter zum Ausgleich unterschiedlicher Varianzen zuvor logarithmiert wurden $(\ln (\mathrm{x}))$. Da es sich bei den untersuchten Parametern um verbundenen Messreihen handelte (Arrhythmieinzidenzen und Aktivitätsniveaus zu unterschiedlichen Zeitpunkten) wurde das Verfahren angepasst ("repeated measurement" ANOVA = univariate Analyse im "randomized blocks design«). Zur Signifikanzprüfung zwischen einzelnen Stichproben innerhalb der ANOVA wurde die post-hoc Testung nach Bonferroni/Dunn durchgeführt. Ein p-Wert $<0,05$ wurde als signifikant gewertet.

\section{Ergebnisse}

\section{Zirkadiane Aktivitätsverteilung}

Die Alltagsaktivitäten der 29 untersuchten Patienten verteilten sich nach Quantifizierung durch das oben erläuterte Punktesystem auf die Tageszeitperioden 1 - 4 wie in Tab.1 dargestellt.

Im Vergleich der zirkadianen Aktivitätskurven (Abb.1) wird der natürliche Tag-Nacht-Rhythmus erkennbar und in den Tagesperioden mit überwiegenden Wachzeit ( = Perioden 2 4:6 Uhr - 23 Uhr 59) eine inverse Verteilung der physischen und mentalen Aktivitäten. Die maximale physische Aktivität wird in den Morgenstunden (Periode 2) und die höchste mentale Aktivität in der Abendzeit (Periode 4) registriert. Bei Addition der physischen und mentalen Aktivitäten

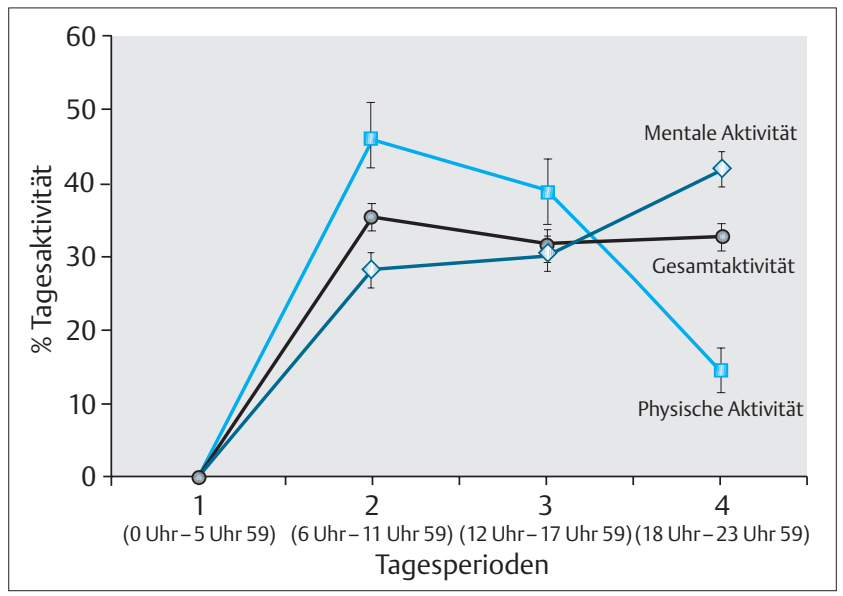

Abb.1 Zirkadiane Verteilung der physischen und mentalen Aktivität. Für "Gesamtaktivität«: ANOVA p <0,0001 und Bonferroni/Dunn $p<0,0001$ für Periode 1 versus 2, 3 und 4. Für »Physische Aktivität»: $p<0,0001$ und Bonferroni/Dunn $p<0,001$ für Periode 2 versus 1 und 4,1 versus 3 und 4 und 3 versus 4. Für »Mentale Aktivität«: $p<0,0001$ und Bonferroni/Dunn $p<0,0001$ für Periode 1 versus 2, 3 und 4 und $p<0,002$ für Periode 4 versus 2 und 3 .

Tab.1 Tageszeitliche Variation der Alltagsaktivitäten

\begin{tabular}{llll}
\multicolumn{4}{l}{ Punktesumme in Tageszeitperiode } \\
1 & 2 & 3 & 4 \\
(0 Uhr bis & (6 Uhr bis & (12 Uhr bis & (18 Uhr bis \\
5 Uhr 59) & 11 Uhr 59) & 17 Uhr 59) & 23 Uhr 59)
\end{tabular}

\section{Physische}

Aktivitäten

Minimum -

Maximum

Median

$\begin{array}{ll}0,2 \pm 1,1 & 7 \pm 5 \\ 0-6 & 0-19\end{array}$

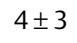

$2,5 \pm 4$

0

6

$0-15$

$0-12,5$

Mentale

Mittelwert+ SD

Minimum -

Maximum

Median

$0-0$

$6 \pm 3$

$7 \pm 4$

$10 \pm 5$

0

$3-12$

$0-13$

$2-21$

Gesamtaktivität

Mittelwert \pm SD

Minimum -

Maximum

Median

\section{$0,2 \pm 1,1 \quad 13 \pm 5$}

$12 \pm 5$

$12 \pm 6$

$0-6$

$3-22,5$

$0,5-23$

$3-31$

0

13,5

11,5

11

Gesamtaktivität $=\sum$ aus physischer und mentaler Aktivität, SD = Standardabweichung

( = Gesamtaktivität) heben sich die Unterschiede in den Tageszeitperioden 2 - 4 weitgehend auf und der Tag-NachtRhythmus wird prononciert.

\section{Zirkadiane Arrhythmieverteilung}

Die gemittelte tageszeitliche Verteilung der Rezidivarrhythmien ( $44 \pm 58$; Median 28; Minimum 4, Maximum 235 pro Patient) der 29 Patienten während einer Nachbeobachtungszeit von $59 \pm 28$ Monaten (Minimum 6, Maximum 100) entspricht dem typischen Muster mit Häufigkeitsgipfel in den Morgenstunden (Abb.2). Die Anzahl der Arrhythmierezidive war bei Patienten mit ausgeprägterer Einschränkung der 


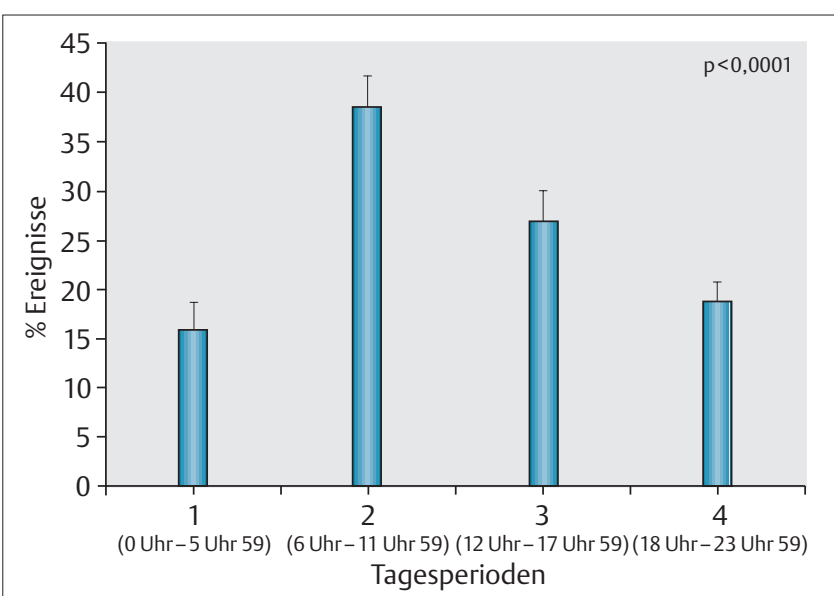

Abb.2 Zirkadiane Rezidivverteilung (531 Ereignistage; $18 \pm 20$ pro Patient; Median 11; Minimum 3, Maximum 99 Ereignistage). Bonferroni/Dunn $\mathrm{p}<$ 0,001 für Periode 2 versus 1 und 4, $p<0,008$ für Periode 3 versus 2 und 1 .

linksventrikulären Pumpfunktion nicht signifikant höher als bei den übrigen (mittlere Ereignisrate pro Monat 0,9 $\pm 0,8$ median 0,8 vs $0,6 \pm 0,8$ median 0,4 bei zwölf Patienten mit LVEF $\leq 30 \%$ vs 17 Patienten mit LVEF > 30\%).

Bei 25 Patienten (86\%) war eine einzelne Tageszeitperiode mit Ereignisgipfel zu benennen, die bei der Mehrzahl dieser Patienten ( 22 von $25=88 \%$ ) in der Wachzeit lag: in Tageszeitperiode 2 bei 15 Patienten (60\%), in Periode 3 bei vier Patienten $(16 \%)$ und in Periode 4 bei drei Patienten (12\%). Bei drei Patienten (12\%) wurden die meisten Rezidive während der Schlafzeit registriert (Tab.2).

Bei einem Vergleich der Ereignishäufigkeit über Tag und in der Nacht spiegelt sich der natürliche Schlaf-Wach-Rhythmus in Form einer etwa doppelt so hohen Ereignishäufigkeit über Tag wider (Tab.3).

\section{Vergleich der zirkadianen Aktivitäts- und Arrhythmieverteilung}

Möglichen Auswirkungen von Aktivitätsunterschieden auf die Rezidivhäufigkeit innerhalb der Wachzeit zeigt ein Vergleich der tageszeitlichen Maxima von Rezidivhäufigkeit und Aktivität in den Tageszeitperioden 2 - 4: Bei Analyse der Gesamtaktivität fand sich bei zehn von 22 Patienten mit benennbarem tageszeitlichen Rezidivgipfel (45\%) die höchste Aktivität zur Tageszeit mit den meisten Rezidiven. Bei Betrachtung der physischen und mentalen Aktivitäten alleine traf dies für zwölf Patienten (55\%) bzw. 5 Patienten (23\%) zu.

Ein Vergleich des Aktivitätsniveaus in der Tagesperiode mit den häufigsten Ereignissen und dem mittleren Aktivitätsniveau in den übrigen Tagesperioden ergab signifikant höhere Werte für die physische Aktivität in dem Tageszeitraum mit individuellem Ereignisgipfel (Tab.4).

Bei linearer Regression der in Tab.2 abgebildeten Prozentverteilung der Alltagsaktivität (unabhängige Variable) gegen den prozentualen Anteil an Rezidiven im Langzeitverlauf (abhängige Variable) zeigt sich eine positive Korrelation zwischen dem Ausmaß der gewohnheitsmäßigen Aktivität und der Anzahl der in der entsprechenden Tagesperiode erlebten Rezidivar- rhythmien sowohl für die Gesamtaktivität als auch für die physische und mentale Aktivität. Bei Betrachtung der Wachphase alleine (Tagesperioden $2-4$ ) bleibt die positive Korrelation für die Gesamtaktivität und physische Aktivität erhalten.

Nach einem Paarvergleich der individuellen Steigungswerte gegen Null ist die positive Korrelation zwischen Aktivität und Arrhythmiehäufigkeit unter Einschluss aller vier Tageszeitperioden erwartungsgenäß signifikant für die Gesamtaktivität ( $\mathrm{p}<$ 0,008 , Steigung $>0$ bei $75 \%$ der Patienten), für die physische Aktivität ( $p<0,003$, Steigung $>0$ bei $70 \%$ der Patienten) und für die mentale Aktivität ( $p<0,03$, Steigung $>0$ bei $64 \%$ der Patienten). Bei Beschränkung der Analyse auf die Wachzeitperioden 2 - 4 bleibt der Test für die Gesamtaktivität signifikant ( $p<0,05$, Steigung $>0$ bei $67 \%$ der Patienten); für die physische Aktivität verfehlt er das Signifikanzniveau nur knapp $(p=0,056$, Steigung $>0$ bei $56 \%$ der Patienten) und ist für die mentale Aktivität nicht signifikant ( $p=0,84$, Steigung $>0$ bei $46 \%$ der Patienten).

\section{Diskussion}

Die vergleichbaren tageszeitlichen Verteilungsmuster des plötzlichen Herztodes und ventrikulärer Tachyarrhythmien $(6,13,20,28)$ legen nahe, dass diese kardiovaskulären Ereignisse durch ähnliche Triggersituationen beeinflusst werden. Alltägliche physische und mentale Belastungen sind als Trigger myokardischämischer Ereignisse erkannt worden $(2,4,5,8,11,12,16,17)$. Daher stellt sich die Frage, ob nicht auch ventrikuläre Tachyarrhythmien durch Alltagsbelastungen getriggert werden können. Als plausibles pathophysiologisches Bindeglied zwischen Aktivität und Arrhythmie kommen die aktivitätsassoziierte sympathoadrenerge Aktivierung und transiente Myokardischämien in Frage. Die vorliegenden Untersuchungsergebnisse machen erstmals einen Zusammenhang zwischen der tageszeitlichen Häufigkeitsverteilung von Rezidivarrhythmien bei Defibrillatorträgern und der Verteilung gewohnter Aktivitäten wahrscheinlich und bekräftigen so, dass Arrhythmien durch Alltagsaktivitäten getriggert werden können.

Bereits der in der zirkadianen Rezidivverteilung deutlich erkennbare Tag-Nacht-Rhythmus spricht für eine teilweise Aktivitätsabhängigkeit der dokumentierten ventrikulären Tachyarrhythmien bei den untersuchten Patienten. Der mittlere Anteil von über Tag registrierten Ereignissen pro Zeiteinheit erwies sich als nahezu doppelt so hoch wie der von nächtlichen Arrhythmien (s. Tab.3). Übereinstimmend hierzu zeigte die Analyse der individuellen Ereignisgipfel die höchste Rezidivinzidenz in den Nachtstunden nur bei $10 \%$ der Patienten. Die positive Korrelation zwischen der Tag-Nacht-Aktivität und der zirkadianen Arrhythmieverteilung wird auch erkennbar bei der linearen Regression der mittleren Aktivitätsniveaus in allen vier Tageszeitperioden gegen den entsprechenden Anteil an Rezidivereignissen.

Über die durch den natürlichen Tag-Nacht-Rhythmus bedingte Verteilung von Aktivität und Ruhe hinaus scheinen sich jedoch auch Unterschiede in der habituellen Aktivitätsverteilung während der überwiegenden Wachphase (Tageszeitperiode 2 - 3 = 6 Uhr bis 23 Uhr 59) auf die Häufigkeitsverteilung der Rezidivarrhythmien in diesem Tageszeitraum auszuwirken. Bei einem Vergleich der individuellen tageszeitlichen Rezidivgipfel mit den individuellen Aktivitätsmaxima in den Tageszeitperioden 2 - 4 wäre unter der Voraussetzung, dass 
Tab.2 Prozentuale zirkadiane Verteilung der Arrhythmierezidive und der Gewohnheitsaktivitäten (tageszeitliche Maximalwerte fett gedruckt).

\begin{tabular}{|c|c|c|c|c|c|c|c|c|c|c|c|c|c|c|c|c|c|}
\hline \multirow[b]{2}{*}{ Fall } & \multicolumn{4}{|c|}{ \% $\mathrm{E}$ in TZP } & \multirow{2}{*}{$\begin{array}{l}\text { Einzelne T mit } \\
\text { E-Max. in TZP mit } \\
\text { mittl. E-Max. }\end{array}$} & \multicolumn{4}{|c|}{$\begin{array}{l}\text { \% Physische } \\
\text { Aktivität in TZP }\end{array}$} & \multicolumn{4}{|c|}{$\begin{array}{l}\text { \% Mentale } \\
\text { Aktivität in TZP }\end{array}$} & \multicolumn{4}{|c|}{ \% Gesamtaktivität in TZP } \\
\hline & 1 & 2 & 3 & 4 & & 1 & 2 & 3 & 4 & 1 & 2 & 3 & 4 & 1 & 2 & 3 & 4 \\
\hline 1 & 44 & 23 & 17 & 16 & $10 / 28(36 \%)$ & 0 & 78 & 22 & 0 & 0 & 27 & 29 & 44 & 0 & 39 & 27 & 34 \\
\hline 2 & 50 & 20 & 5 & 25 & $2 / 4(50 \%)$ & 0 & 63 & 29 & 8 & 0 & 16 & 16 & 68 & 0 & 42 & 23 & 35 \\
\hline 3 & 43 & 27 & 7 & 23 & $13 / 30(43 \%)$ & 0 & 31 & 41 & 28 & 0 & 43 & 24 & 33 & 0 & 36 & 33 & 31 \\
\hline 4 & 25 & 46,5 & 7 & 21,5 & $13 / 28(46 \%)$ & 0 & 52 & 48 & 0 & 0 & 22 & 39 & 39 & 0 & 31 & 42 & 27 \\
\hline 5 & 10,5 & 44 & 36,5 & 9 & $5 / 11(45 \%)$ & 0 & 44 & 23 & 33 & 0 & 37,5 & 37,5 & 25 & 0 & 42 & 26 & 32 \\
\hline 6 & 25 & 32 & 15 & 28 & $3 / 8(38 \%)$ & 0 & 52 & 48 & 0 & 0 & 54 & 0 & 46 & 0 & 53 & 22 & 25 \\
\hline 7 & 7 & 43 & 20 & 30 & $13 / 30(43 \%)$ & 0 & 21 & 79 & 0 & 0 & 13 & 35 & 52 & 0 & 15 & 45 & 40 \\
\hline 8 & 19,5 & 56 & 19,5 & 7 & $15 / 27(56 \%)$ & 0 & 61 & 31 & 8 & 0 & 26 & 35 & 39 & 0 & 34 & 34 & 32 \\
\hline 9 & 13 & 44 & 27 & 16 & $14 / 33(42 \%)$ & 0 & 0 & 75 & 25 & 0 & 27 & 35 & 38 & 0 & 23 & 40 & 37 \\
\hline 10 & 6 & 64 & 16 & 12 & $60 / 99(61 \%)$ & 0 & 38 & 56 & 6 & 0 & 44 & 25 & 31 & 0 & 42 & 33 & 25 \\
\hline 11 & 0 & 45 & 41 & 14 & $10 / 22(45 \%)$ & 0 & 60 & 40 & 0 & 0 & 15 & 51 & 34 & 0 & 24 & 49 & 27 \\
\hline 12 & 14 & 58 & 14 & 14 & $7 / 7(100 \%)$ & 23 & 50 & 19 & 8 & 0 & 11 & 11 & 78 & 11 & 30 & 15 & 44 \\
\hline 13 & 19 & 47 & 15 & 19 & $7 / 16(44 \%)$ & 0 & 58 & 42 & 0 & 0 & 33 & 33,5 & 33,5 & 0 & 41 & 36 & 23 \\
\hline 14 & 25 & 50 & 25 & 0 & $2 / 4(50 \%)$ & 0 & 44 & 0 & 56 & 0 & 48 & 4 & 48 & 0 & 47 & 2 & 51 \\
\hline 15 & 0 & 50 & 33 & 17 & $3 / 6(50 \%)$ & 0 & 90 & 10 & 0 & 0 & 28 & 31 & 41 & 0 & 43 & 26 & 31 \\
\hline 16 & 33 & 67 & 0 & 0 & $2 / 3(67 \%)$ & 0 & 44 & 22 & 34 & 0 & 17 & 33 & 50 & 0 & 30 & 28 & 42 \\
\hline 17 & 0 & 68 & 32 & 0 & $4 / 6(67 \%)$ & 0 & 33 & 38 & 29 & 0 & 18 & 53 & 29 & 0 & 24 & 47 & 29 \\
\hline 18 & 0 & 60 & 20 & 20 & $3 / 5(60 \%)$ & 0 & 83 & 0 & 17 & 0 & 30 & 40 & 30 & 0 & 39 & 33 & 28 \\
\hline 19 & 10 & 33 & 35 & 22 & $7 / 20(35 \%)$ & 0 & 0 & 60 & 40 & 0 & 38 & 6 & 56 & 0 & 32 & 14 & 54 \\
\hline 20 & 21 & 14 & 43 & 22 & $14 / 32(44 \%)$ & 0 & 45 & 33 & 22 & 0 & 18 & 55 & 27 & 0 & 30 & 45 & 25 \\
\hline 21 & 0 & 40 & 60 & 0 & $3 / 5(60 \%)$ & 0 & 0 & 100 & 0 & 0 & 40 & 36 & 24 & 0 & 35 & 44 & 21 \\
\hline 22 & 25 & 0 & 75 & 0 & $3 / 4(75 \%)$ & 0 & 50 & 33 & 17 & 0 & 24 & 37,5 & 37,5 & 0 & 31 & 37 & 32 \\
\hline 23 & 0 & 17 & 33 & 50 & $3 / 6(50 \%)$ & 0 & 48 & 11 & 41 & 0 & 17 & 29 & 54 & 0 & 33 & 20 & 47 \\
\hline 24 & 4 & 32 & 27 & 37 & $13 / 38(34 \%)$ & 0 & 58 & 32 & 10 & 0 & 25 & 25 & 50 & 0 & 44 & 29 & 27 \\
\hline 25 & 0 & 29 & 25 & 46 & $5 / 12(42 \%)$ & 0 & 40 & 60 & 0 & 0 & 38 & 25 & 38 & 0 & 38 & 31 & 31 \\
\hline 26 & 25 & 30 & 30 & 15 & - & 0 & 67 & 33 & 0 & 0 & 28,5 & 28,5 & 43 & 0 & 37 & 30 & 33 \\
\hline 27 & 25 & 25 & 25 & 25 & - & 0 & 40 & 60 & 0 & 0 & 38 & 24 & 38 & 0 & 38 & 30 & 32 \\
\hline 28 & 33 & 9 & 25 & 33 & - & 0 & 0 & 0 & 0 & 0 & 33 & 33,5 & 33,5 & 0 & 33 & 34 & 33 \\
\hline 29 & 0 & 40 & 40 & 20 & - & 0 & 44 & 35 & 21 & 0 & 17 & 44 & 39 & 0 & 36 & 38 & 26 \\
\hline
\end{tabular}

$\mathrm{E}=$ Ereignis, $\mathrm{T}=$ Ereignistag, E-Max. $=$ Ereignismaximum, TZP $=$ Tageszeitperiode

zwischen der Verteilung der Tagesaktivitäten und der zirkadianen Variation der Rezidivarrhythmien kein Zusammenhang besteht, ein Zusammentreffen des tageszeitlichen Aktivitätsmaximums mit dem individuellen Rezidivgipfel in 25\% der Patienten zu erwarten (entsprechend den vier Möglichkeiten der Verteilung des Aktivitätsmaximums auf Tageszeitperiode 2, 3, 4 oder einer gleichmäßigen Aktivitätsverteilung). Tatsächlich fand sich jedoch eine Übereinstimmung bezüglich der Gesamtaktivität bei $45 \%$ und bezüglich der physischen Aktivität sogar bei 55\% der Patienten. Die Annahme eines dominierenden Einflusses physischer Aktivitäten wird durch den Vergleich der mittleren Aktivitätsanteile pro Zeiteinheit in der Tageszeitperiode mit Ereignisgipfel und den anderen Tagesperioden bestätigt (s. Tab.4). Hier findet sich allein für die physische Aktivität ein signifikant höherer Anteil in dem Tageszeitraum mit den meisten Rezidiven. Bei Analyse der linearen Regression der Aktivität gegen die Arrhythmiehäufigkeit während der Wachphase verfehlt die positive Korrelation zwischen Rezidivhäufigkeit und physischer Aktivitätsverteilung dementsprechend nur knapp das Signifikanzniveau $(p=0,056)$. Der Nachweis einer signifikanten Korrelation zwischen der Aktivitäts- und Arrhythmieverteilung bei Berück- sichtigung der Summe aus physischen und mentalen Aktivitäten kann als Beitrag der mentalen Aktivitätsverteilung zur positiven Korrelation zwischen Aktivitätsniveau und Ereignishäufigkeit diskutiert werden.

An dieser Stelle ist auf methodische Limitationen der Datenerfassung hinzuweisen. Aussagen nach eigenanamnestischen Angaben über den Grad der physischen und mentalen Belastung gründen in den bisher zu dieser Thematik durchgeführten nicht experimentellen Untersuchungen auf Tagebuchdokumentation der Patienten $(2,11,12,14,16,17)$ oder wurden durch Befragung gewonnen (5, 10, 18, 19,24-26). Hierbei kamen zahlreiche Verfahren zur Datenerfassung und Quantifizierung von physischen und mentalen Belastungen zur Anwendung. Eine klare Trennung zwischen mentaler und physischer Belastung des Organismus ist jedoch nicht möglich, da einerseits jede physische Tätigkeit auch ein wechselnd hohes $\mathrm{Ma}$ an mentaler Aktivität erfordert, und andererseits viele mentale Tätigkeiten (z.B. Autofahren, Computer- und Handarbeiten, Gesellschaftsspiele) von physischer Aktivität begleitet sind. Das Ausmaß von körperlicher Aktivität ist in der Selbstbeobachtung zudem einfacher zu begreifen und kann in 
Tab.3 Tag-Nacht-Verteilung der Rezidivarrhythmien und der Gewohnheitsaktivität.

\begin{tabular}{|c|c|c|c|}
\hline & $\begin{array}{l}\text { Tag } \\
\text { (6 Uhr - } 23 \text { Uhr 59) }\end{array}$ & $\begin{array}{l}\text { Nacht } \\
\text { (24 Uhr - } 5 \text { Uhr 59) }\end{array}$ & p \\
\hline $\begin{array}{l}\text { Rezidive } \\
\text { (\% pro } 6 \text { Stunden) }\end{array}$ & $28 \pm 5$ & $16 \pm 15$ & $<0,01$ \\
\hline $\begin{array}{l}\text { Physische Aktivität } \\
\text { (\% pro } 6 \text { Stunden) }\end{array}$ & $32 \pm 6$ & $0,8 \pm 4$ & $<0,0001$ \\
\hline $\begin{array}{l}\text { Mentale Aktivität } \\
\text { (\% pro } 6 \text { Stunden) }\end{array}$ & $33 \pm 0$ & $0 \pm 0$ & $<0,0001$ \\
\hline $\begin{array}{l}\text { Gesamtaktivität } \\
\text { (\% pro 6 Stunden) }\end{array}$ & $33 \pm 1$ & $0,4 \pm 2$ & $<0,0001$ \\
\hline
\end{tabular}

Tab.4 Niveau der Alltagsaktivität zur Tageszeit mit der individuell höchsten Ereignishäufigkeit im Vergleich zum Aktivitätsniveau in den übrigen Tageszeitperioden.

\begin{tabular}{llll}
\hline & $\begin{array}{l}\text { Tageszeitperiode } \\
\text { mit Ereignisgipfel }\end{array}$ & $\begin{array}{l}\text { Übrige } \\
\text { Tageszeitperioden }\end{array}$ & $\mathrm{P}$ \\
\hline $\begin{array}{l}\text { Physische Aktivität } \\
\text { (\% pro 6 Stunden) }\end{array}$ & $47 \pm 25$ & $26 \pm 13$ & $<0,05$ \\
$\begin{array}{l}\text { Mentale Aktivität } \\
\text { (\% pro 6 Stunden) }\end{array}$ & $32 \pm 15$ & $34 \pm 7$ & 0,14 \\
$\begin{array}{l}\text { Gesamtaktivität } \\
\text { (\% pro 6 Stunden) }\end{array}$ & $35 \pm 10$ & $32 \pm 5$ & 0,87 \\
\hline
\end{tabular}

einer Befragung zu dem Gewohnheitsverhalten wahrscheinlich mit größerer Präzision wiedergegeben werden.

Die untersuchten Patienten stellen ein kleineres Subkollektiv einer Gesamtgruppe von Defibrillatorpatienten eines Zentrums dar. Eine Verfälschung der Untersuchungsergebnisse erscheint hieraus jedoch nicht ableitbar, da die klinischen Charakteristika der untersuchten Gruppe im Vergleich mit anderen Studien denen eines typischen Kollektivs von Defibrillatorträgern entsprechen.

\section{Folgerung}

Die vorliegende Untersuchung liefert eine plausible Erklärung für die vielzitierte tageszeitliche Variation von kardiovaskulären Krankheitsereignissen. Eine Bestätigung der Ergebnisse in einer größeren Patientengruppe vorausgesetzt kann diskutiert werden, ob die tageszeitliche Variation kardiovaskulärer Krankheitsereignisse nicht maßgeblich durch die habituelle tageszeitliche Verteilung von Alltagsaktivitäten bestimmt ist. Der Nachweis eines Zusammenhangs zwischen Aktivitätsund Arrhythmieverteilung könnte demnach im Einzelfall Grundlage für eine Umstellung der medikamentösen Therapie bzw. Veränderung der Gewohnheitsaktivitäten sein und so möglicherweise $\mathrm{zu}$ einer Verhinderung schmerzhafter Schockabgaben bei Defibrillatorträgern beitragen.

\section{Literatur}

1 Baecke JAH, Burema J, Frijters JER. A short questionnaire for the measurement of habitual physical activity in epidemiological studies. Am Clin Nutr 1982; 36: 936-942

2 Barry J, Selwyn AP, Nabel EG et al. Frequency of ST-segment depression produced by mental stress in stable angina pectoris from coronary artery disease. Am J Cardiol 1988; 61: 989-993

3 Bayes de Luna A, Coumel P, Leclercq JF. Ambulatory sudden cardiac death: mechanisms of production of fatal arrhythmia on the basis of data from 157 cases. Am Heart J 1989; 117: 151-159
4 Boltwood MD, Taylor CB, Burke MB, Grogin H, Giacomini J. Anger report predicts coronary artery vasomotor response to mental stress in atherosclerotic segments. Am J Cardiol 1993; 72: 1361-1365

5 Burg MM, Jain D, Soufer R, Kerns RD, Zaret BL. Role of behavioral and psychological factors in mental-stress induced silent left ventricular dysfunction in coronary artery disease. J Am Coll Cardiol 1993: 22: 440-448

6 Cohen MC, Rohtla KM, Lavery CE, Muller JE, Mittleman MA. Meta-analysis of the morning excess of acute myocardial infarction and sudden cardiac death. Am J Cardiol 1997; 79: 1512-1516

7 Cottington EM, Matthews KA, Talbott E, Kuller LH. Environmental events preceding sudden death in women. Psychosom Med 1980; 42: 567-574

8 Deanfield JE, Shea M, Kensett $M$ et al. Silent myocardial ischemia due to mental stress. Lancet 1984; 2: 1001-1005

9 Eliot RS, Buell JC. Role of emotions and stress in the genesis of sudden death. J Am Coll Cardiol 1985; 5: 95B-98B(suppl 1)

10 Frasure-Smith N, Lesperance F, Talajic M. Depression and 18-month prognosis after myocardial infarction. Circulation 1995; 91: 999-1005

11 Freedman SB, Wong CK. Triggers of daily life ischemia. Heart 1998; 80 489-492

12 Freeman LJ, Nixon PGF, Sallabank P, Reaveley D. Psychological stress and silent myocardial ischemia. Am Heart J 1987; 114: 477-482

13 Fries R, Heisel A, Jung J, Stopp M, Schieffer H, Özbek C. Tageszeitliche Verteilung von spontanen ventrikulären Tachyarrhythmien bei Patienten mit implantierbarem Kardioverter-Defibrillator. Z Kardiol 1996; 85: 140-147

14 Gabbay FH, Krantz DS, Willem JK et al. Triggers of myocardial ischemia during daily life in patients with coronary artery disease: physical and mental activities, anger and smoking. J Am Coll Cardiol 1996; 27: 585-592

15 Glass RI, Jr. Zack MM. Increase in death from ischemic heart disease after blizzards. Lancet 1979; 1: 485-487

16 Gottdiener JS, Krantz DS, Howell RH et al. Induction of silent myocardial ischemia with mental stress testing: relationship to the triggers of ischemia during daily life activities and to ischemic functional severity. J Am Coll Cardiol 1994; 24: 1645-1651

17 Gullette ECD, Blumenthal JA, Babyak M et al. Effects of mental stress on myocardial ischemia during daily life. JAMA 1997; 277: 1521-1526

18 Hermann C, Riedemann C, Bergmann G et al. Präoperative Angst und Depressivität als unabhängige Prädiktoren von DC-Schocks in den ersten drei Monaten nach ICD-Implantation. Z Kardiol (abstract) 1998; 87: 65(Supp 1

19 Lampe F, Brüggemann T, Ehlers C, Wegscheider K, Andresen D. Factors triggering appropriate shock episodes in patients with an implantable defibrillator. Eur Heart J (abstract) 1997; 18: 97

20 Lampert R, Rosenfeld L, Batsford W, Lee F, McPherson C. Circadian variation of sustained ventricular tachycardia in patients with coronary artery disease and implantable cardioverter defibrillator. Circulation 1994; 90: 241-247

21 Leor J, Poole WK, Kloner RA. Sudden cardiac death triggered by an earthquake. N Engl J Med 1996; 334: 413-419

22 Marti B, Goerre S, Spuhler T, Schaffner T, Gutzwiller F. Sudden death during mass running events in Switzerland 1978-1987: an epidemiologico-pathologic study. Schweiz Med Wochenschr 1989; 119: 473-482

23 Meisel SR, Kutz I, Dayan KI et al. Effect of Iraqui missile war on incidence of acute myocardial infarction and sudden death in Israeli civilians. Lancet 1991; 338: 660-661

24 Mittleman MA, Maclure M, Sherwood JB et al. Triggering of acute myocardial infarction onset by episodes of anger. Determinants of Myocardial Infarction Onset Study Investigators. Circulation 1995; 92: 1720-1725

25 Mittleman MA, Maclure M, Tofler GH, Sherwood JB, Goldberg RJ, Muller JE. Triggering of acute myocardial infarction by heavy physical exertion. Protection against triggering by regular exertion. Determinants of Myocardial Infarction Onset Study Investigators. N Engl J Med 1993; 329: 1677-1683

26 Mittleman MA, Maclure M, Sherwood JB et al. Triggering of acute myocardial infarction onset by episodes of anger. Determinants of Myocardial Infarction Onset Study Investigators. Circulation 1995; 92: 1720-1725

27 Moritz AR, Zamcheck M. Sudden and unexpected deaths of young soldiers: diseases responsible for such deaths during World War II. Arch Pathol 1946; 42: 459-494

28 Tofler GH, Gebara OCE Mittleman MA et al for the CPI Investigators. Morning peak in ventricular tachyarrhythmia detected by time of implantable cardioverter/defibrillator therapy. Circulation 1995; 92: 1203-1208

29 Verrier RL, Hagestad EL, Lown B. Delayed myocardial ischemia induced by anger. Circulation 1987: 75: 249-254

30 Willich SN, Goldberg RJ, Maclure M, Perriello L, Muller JE. Increased onset of sudden cardiac death in the first three hours after awakening. Am J Cardiol 1992; 70: 65-68

31 Yamamoto K, Ikeda U, Fukazawa H, Shimada K. Circadian variation in the incidence of cardioembolism. Am J Cardiol 1996; 78: 1312-1314

\section{Korrespondenz}

Priv.-Doz. Dr. Roland Fries

Innere Medizin III, Kardiologie/Angiologie

Universitätskliniken des Saarlandes

66421 Homburg/Saar

Tel.: 06841/1623016

Fax: 06841/1623369

E-Mail: fries@med-in.uni-sb.de 